\title{
A Case of Synchronous Primary Gastric Cancer and Primary Hepatocellular Carcinoma
}

\begin{abstract}
Synchronous occurrence of primary gastric cancer and hepatocelluar carcinoma is very rare. It is mandatory to differentiate synchronous two primary lesions from metastatic lesion, because treatment modality is different for each disease. We report an interesting case of double malignancy, synchronous primary gastric cancer, and primary hepatocelluar carcinoma, who never had a cirrhosis, family history of cancer as well as hepatitis B or hepatitis $\mathrm{C}$ infection. Our patient managed with palliative chemotherapy due to multicentric hepatocelluar carcinoma with primary gastric cancer.
\end{abstract}

Keywords: Gastric carcinoma, hepatocellular carcinoma, palliative chemotherapy, synchronous malignancy

\section{Introduction}

Synchronous double malignancies involving different organs are relatively rare compared to metastatic lesions. The incidence of gastric cancer with a synchronous second primary cancer varies from $2.0 \%$ to $10.9 \%{ }^{[1]}$ The most common second primary associated with gastric cancer was colorectal, followed by hepatocellular carcinoma, renal cell carcinoma, and pancreatic adenocarcinoma. ${ }^{[2]}$ It is mandatory to differentiate synchronous two primary lesions from the metastatic lesion for the treatment purpose. Here, a case of synchronous primary gastric cancer along with primary multicentric hepatocellular carcinoma was treated with palliative chemotherapy.

\section{Case Report}

A 46-year-old female patient admitted with a complaint of abdominal pain for 3 months duration, gradual onset, progressive, and dull aching with history of nausea and vomiting for 3 months duration. There was a history of loss of appetite and loss of weight. No history of hematemesis, early satiety, and melanoma. No history of the ball rolling movement or abdominal distension. No family history of malignancies. Abdominal examination showed irregular mass present in the epigastric and right hypochondrial

This is an open access journal, and articles are distributed under the terms of the Creative Commons Attribution-Non Commercial-ShareAlike 4.0 License, which allows others to remix, tweak, and build upon the work non-commercially, as long as appropriate credit is given and the new creations are licensed under the identical terms.

For reprints contact: reprints@medknow.com region which moves with respiration and continuous with liver border. Provisional diagnosis made as liver mass for evaluation.

Investigations showed anemia with normal renal function test and liver function test. Serum viral markers for hepatitis $\mathrm{B}$ virus, hepatitis $\mathrm{C}$ virus negative. Coagulation profile was normal. Ultrasound abdominal examination showed enlarged liver measuring $28 \mathrm{~cm}$ with multiple hypoechoic solid lesions present in the liver, replacing the whole liver [Figure 1]. A provisional diagnosis is multicentric hepatocellular carcinoma or metastasis. Hence, we proceeded with serum alpha-fetoprotein which is $26,835 \mathrm{ng} / \mathrm{ml}$ (normal range $0.89-8.78 \mathrm{ng} / \mathrm{ml}$ ). Lower gastrointestinal (GI) endoscopy was normal. Upper GI endoscopy showed ulceroproliferative growth present in antropyloric region and biopsy was taken [Figure 2]. Biopsy was poorly differentiated adenocarcinoma arising from gastric mucosa [Figure 3]. Triple phase contrast CT showed the presence of arterial enhancement followed by delayed hypo intensity of the tumor in the portal venous and delayed phases with antropyloric growth with perigastric lymphadenopathy. Provisional diagnosis based on the triple phase computed tomography was multicentric hepatocellular carcinoma with antropyloric growth [Figure $4 \mathrm{a}$ and $\mathrm{b}$ ]. To differentiate alpha-fetoprotein producing

How to cite this article: Pandiaraja J. A case of synchronous primary gastric cancer and primary hepatocellular carcinoma. Indian J Med Paediatr Oncol 2018;39:405-7.
Jayabal Pandiaraja

Department of General Surgery, SRM Medical College, Kancheepuram, Tamil Nadu, India

Address for correspondence: Dr. Jayabal Pandiaraja, 26/1, Kaveri Street, Rajaji Nagar, Villivakkam,

Chennai - 600049

Tamil Nadu, India.

E-mail:dr:pandiaraja@gmail. com

Access this article online Website: www.ijmpo.org

DOI: 10.4103/ijmpo.ijmpo_15_17 Quick Response Code:

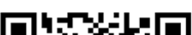
맘ㄴㄷㄴ Hits 1.

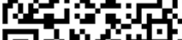
口rits 


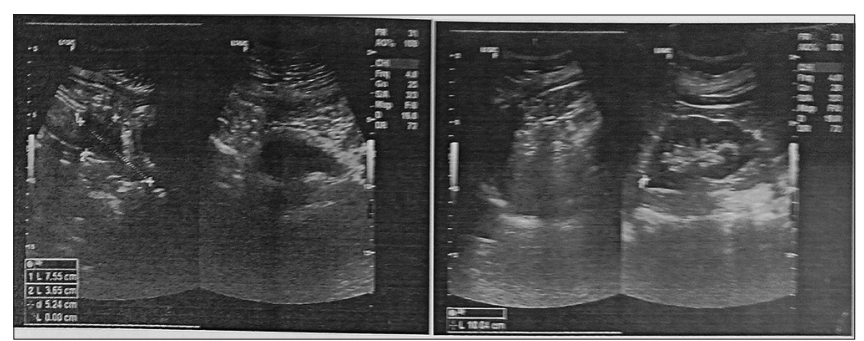

Figure 1: Ultrasound abdominal examination showed enlarged liver measuring $28 \mathrm{~cm}$ with multiple hypo echoic solid lesions present in the liver, replacing the whole liver

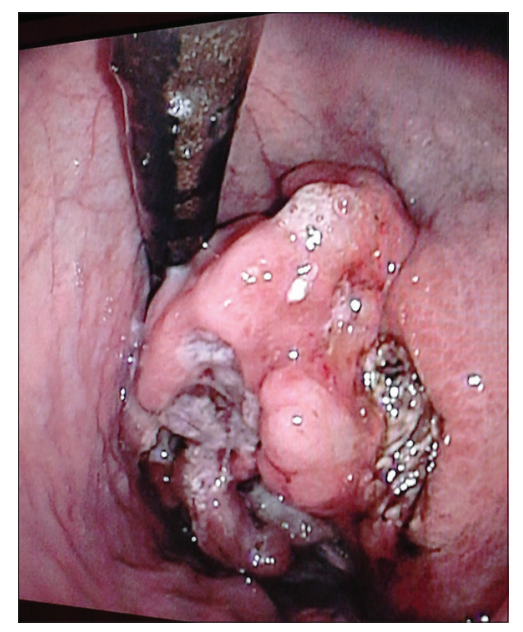

Figure 2: Upper gastrointestinal endoscopy showed ulceroproliferative growth present in antropyloric region and biopsy was taken

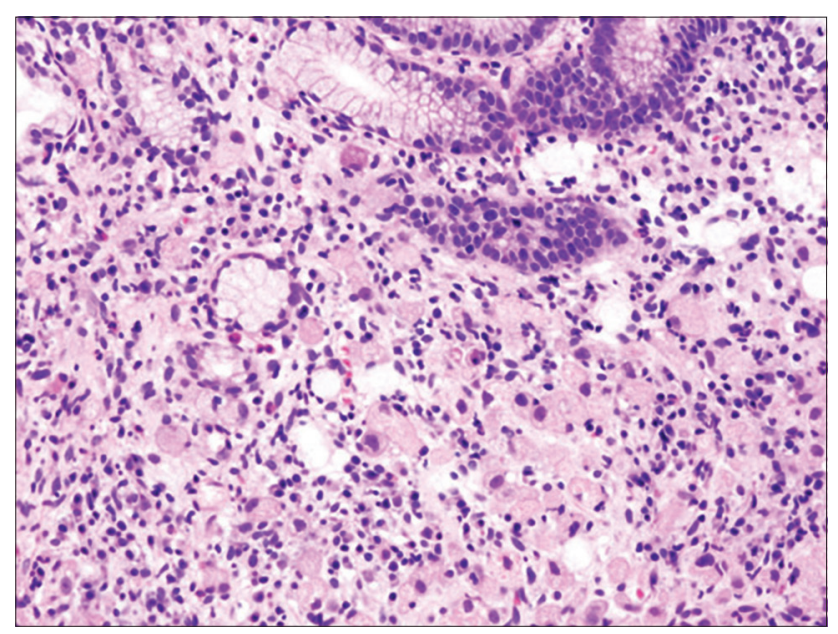

Figure 3: Biopsy was poorly differentiated adenocarcinoma arising from gastric mucosa $(\mathrm{H}$ and $\mathrm{E}, \times 200)$

gastric carcinoma and primary hepatocelluar carcinoma we did fine-needle aspiration cytology with ultrasound guidance. Fine-needle aspiration of the liver mass showed atypical bare nuclei exhibit characteristic hepatocytic nuclear features. Mallory hyaline is seen as reddish clumpy intracytoplasmic material [Figure 5]. Final diagnosis made as synchronous primary gastric cancer with primary multicentric hepatocelluar carcinoma. The patient was treated with combined palliative chemotherapy.

\section{Discussion}

Multiple primary malignant tumors can become synchronous or metachronous depending on the interval between their diagnosis. Synchronous cancer is diagnosed simultaneously or within about 6 months, and metachronous cancers are secondary cancers that developed more than 6 months after the diagnosis and treatment of primary cancer. ${ }^{[3]}$ The criteria of multiple primary tumors include. (1) Each tumor should present a definite picture of malignancy. (2) Each tumor should be histologically distinct. (3) The possibility of metastasis should be excluded from the study.

The etiology of multiple primary malignancies is unknown. However, there are proposed hypothesis such as sharing same carcinogens, childhood chemotherapy or radiotherapy, genetic susceptibility, family history of malignancy, and increased life span. ${ }^{[4]}$ One more hypothesis is field cancerization, which is exposure of carcinogens leads to proliferation of clone of cells. The incidence of synchronous gastric cancer is more with early gastric cancer compared to advanced cancer. In synchronous malignancy, one cancer does not affect the survival of another malignancy when both malignancies resected simultaneously. Extra hepatic primary cancer had no adverse effect on either patient survival or disease-free survival after hepatic resection. ${ }^{[5]}$ The prognosis of early stage gastric cancer patients with a synchronous second primary cancer were influenced more by the presence of the second primary cancer than by gastric cancer itself. ${ }^{[6]}$

Diagnosis of synchronous primary gastric cancer with synchronous primary multicentric hepatocellular carcinoma is difficult without tissue diagnosis. The diagnosis is, even more, difficulty when there is an alpha-fetoprotein producing gastric cancer with solitary primary hepatocellular carcinoma. ${ }^{[7]}$ Since the only investigation confirms diagnosis apart from fine-needle aspiration is alpha-fetoprotein which is elevated in alpha- fetoprotein producing gastric carcinoma.

Treatment for hepatocellular carcinoma includes resection, transplantation and percutaneous ablation with radiofrequency waves or ethanol injection. Liver transplantation is the only curative option when hepatocellular carcinoma is associated with liver disease. ${ }^{[6]}$ Patient with primary gastric cancer with solid hepatocellular carcinoma without cirrhosis or altered liver function can proceed with gastrectomy with hepatectomy ${ }^{[8]}$ It is mandatory to differentiate liver lesion whether primary or metastatic lesion from the second primary. Because solitary liver lesion with operable gastric cancer can be treated curative indent, whereas multiple primary hepatocellular carcinoma or primary gastric cancer with liver metastasis treated by palliative indent. In our case, there are multiple liver lesions which are multicentric hepatocellular carcinoma with primary gastric malignancy. Hence, patient treated with palliative chemotherapy. The most common 

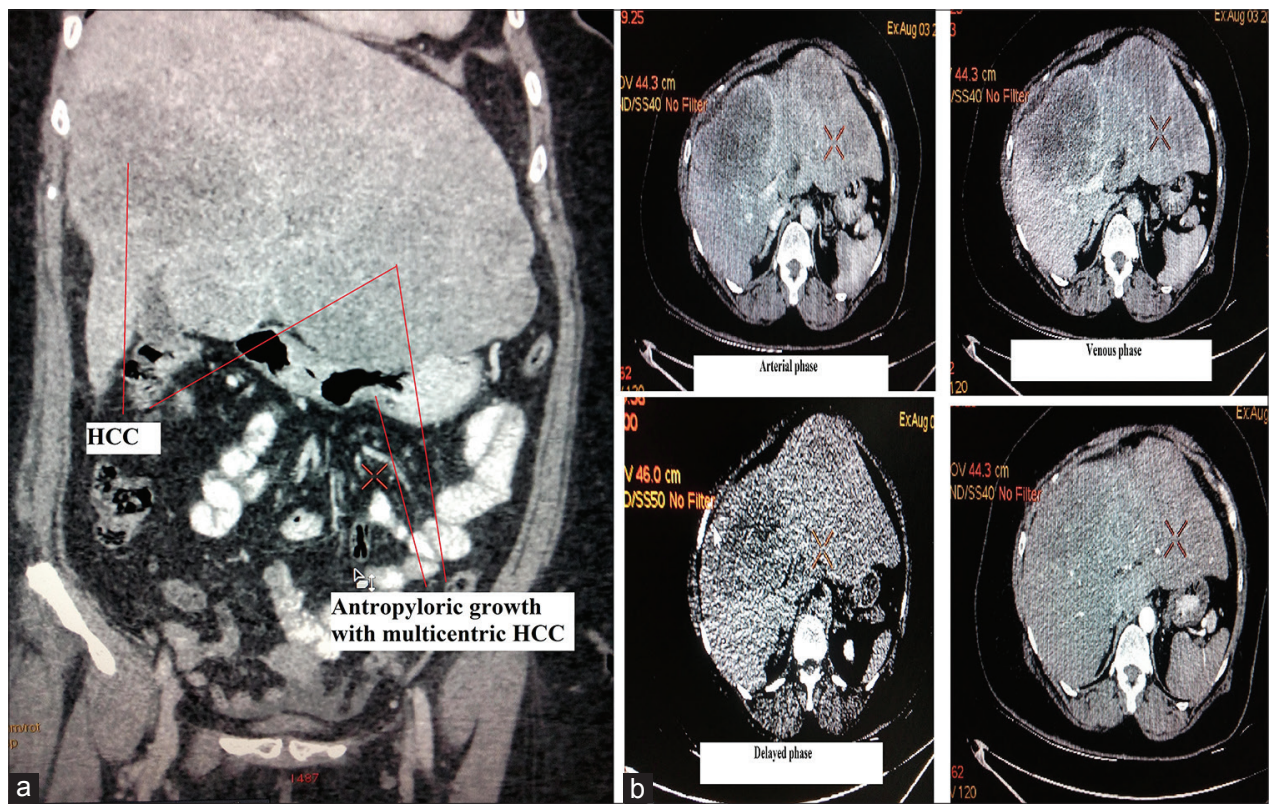

Figure 4: $(a$ and $b)$ Triple phase contrast computed tomography showed presence of arterial enhancement followed by delayed hypo intensity of the tumor in the portal venous and delayed phases with antropyloric growth with perigastric lymphadenopathy

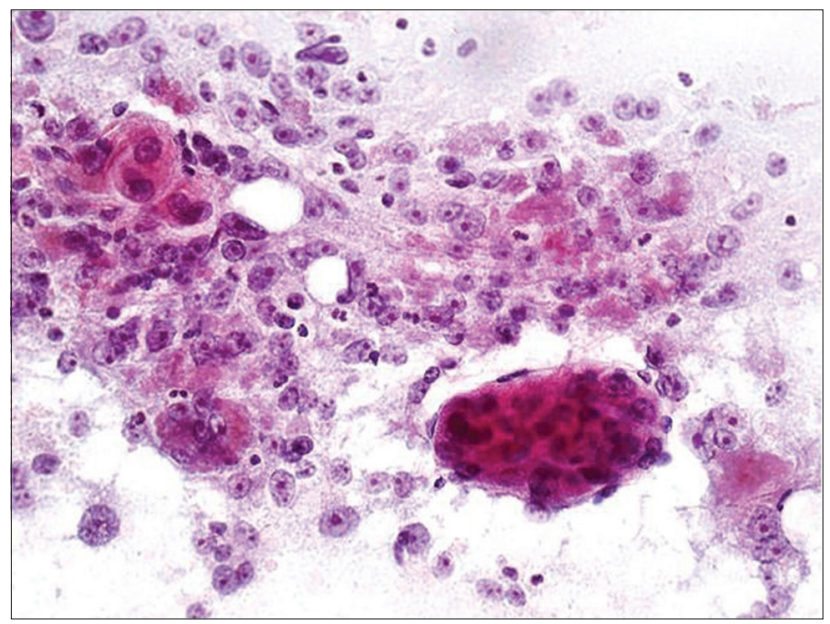

Figure 5: Fine-needle aspiration of liver mass showed atypical bare nuclei exhibit characteristic hepatocytic nuclear features. Mallory hyaline is seen as reddish clumpy intracytoplasmic material (Papanicolaou, $\times 200$ )

cause of death in patient with primary hepatocellular carcinoma and primary gastric cancer is the recurrence of hepatocellular carcinoma. ${ }^{[8]}$

\section{Conclusions}

Synchronous occurrence of primary gastric cancer and hepatocellular carcinoma is very rare. It is mandatory to differentiate synchronous two primary lesions from the metastatic lesion because treatment modality is different for each disease. Even though alpha-fetoprotein rose in hepatocellular carcinoma, it is mandatory to differentiate whether it is produced by alpha feto protein producing gastric carcinoma or hepatocellular carcinoma. Our patient managed with palliative chemotherapy due to multicentric hepatocelluar carcinoma with primary gastric cancer.

Financial support and sponsorship

Nil.

\section{Conflicts of interest}

There are no conflicts of interest.

\section{References}

1. Lee JH, Bae JS, Ryu KW, Lee JS, Park SR, Kim CG, et al. Gastric cancer patients at high-risk of having synchronous cancer. World J Gastroenterol 2006;12:2588-92.

2. Buyukasik O, Hasdemir AO, Gulnerman Y, Col C, Ikiz O. Second primary cancers in patients with gastric cancer. Radiol Oncol 2010;44:239-43.

3. Wang Y, Wu XT. Stomach carcinoma presenting with a synchronous liver cancer: A case report and literature review. Case Rep Gastrointest Med 2014;2014:970293.

4. Chong VH, Idros A, Telisinghe PU. Triple synchronous gastrointestinal malignancies: A rare occurrence. Singapore Med J 2010;51:e176-7.

5. Chang JY, Kim BH, Hong SW, Kim YW, Oh JH. A case report of synchronous double primary liver cancers combined with early gastric cancer. Korean J Intern Med 2003;18:115-8.

6. Ha TK, An JY, Youn HG, Noh JH, Sohn TS, Kim S. Surgical outcome of synchronous second primary cancer in patients with gastric cancer. Yonsei Med J 2007;48:981-7.

7. Kono K, Amemiya H, Sekikawa T, Iizuka H, Takahashi A, Fujii $\mathrm{H}$, et al. Clinicopathologic features of gastric cancers producing alpha-fetoprotein. Dig Surg 2002;19:359-65.

8. Uenishi T, Kubo S, Hirohashi K, Osugi H, Shuto T, Tanaka H, et al. Surgical management of synchronous hepatocellular carcinoma and gastric cancer. Dig Surg 2003;20:133-40. 